\title{
Cost-Effectiveness of a Short Message Service Intervention to Prevent Type 2 Diabetes from Impaired Glucose Tolerance
}

\author{
Carlos K. H. Wong, ${ }^{1}$ Fang-Fang Jiao, ${ }^{1}$ Shing-Chung Siu, ${ }^{2}$ \\ Colman S. C. Fung, ${ }^{1}$ Daniel Y. T. Fong, ${ }^{3}$ Ka-Wai Wong, ${ }^{2}$ Esther Y. T. Yu, ${ }^{1}$ \\ Yvonne Y. C. Lo, ${ }^{1}$ and Cindy L. K. Lam ${ }^{1}$ \\ ${ }^{1}$ Department of Family Medicine and Primary Care, The University of Hong Kong, Ap Lei Chau, Hong Kong \\ ${ }^{2}$ Department of Medicine and Rehabilitation, Tung Wah Eastern Hospital, Causeway Bay, Hong Kong \\ ${ }^{3}$ School of Nursing, The University of Hong Kong, Pokfulam, Hong Kong \\ Correspondence should be addressed to Carlos K. H. Wong; carlosho@hku.hk
}

Received 12 June 2015; Accepted 14 September 2015

Academic Editor: Li-Wei Cho

Copyright (C) 2016 Carlos K. H. Wong et al. This is an open access article distributed under the Creative Commons Attribution License, which permits unrestricted use, distribution, and reproduction in any medium, provided the original work is properly cited.

\begin{abstract}
Aims. To investigate the costs and cost-effectiveness of a short message service (SMS) intervention to prevent the onset of type 2 diabetes mellitus (T2DM) in subjects with impaired glucose tolerance (IGT). Methods. A Markov model was developed to simulate the cost and effectiveness outcomes of the SMS intervention and usual clinical practice from the health provider's perspective. The direct programme costs and the two-year SMS intervention costs were evaluated in subjects with IGT. All costs were expressed in 2011 US dollars. The incremental cost-effectiveness ratio was calculated as cost per T2DM onset prevented, cost per life year gained, and cost per quality adjusted life year (QALY) gained. Results. Within the two-year trial period, the net intervention cost of the SMS group was $\$ 42.03$ per subject. The SMS intervention managed to reduce $5.05 \%$ onset of diabetes, resulting in saving $\$ 118.39$ per subject over two years. In the lifetime model, the SMS intervention dominated the control by gaining an additional 0.071 QALY and saving \$1020.35 per person. The SMS intervention remained dominant in all sensitivity analyses. Conclusions. The SMS intervention for IGT subjects had the superiority of lower monetary cost and a considerable improvement in preventing or delaying the T2DM onset. This trial is registered with ClinicalTrials.gov NCT01556880.
\end{abstract}

\section{Introduction}

Prevention of diabetes mellitus (DM) is becoming an increasing urgent public health concern all over the world. It is estimated that there are 382 million diabetic subjects by 2013 in the world, and the number will increase to 592 million by 2035 [1]. The rising prevalence of DM and its devastating complications [2] poses a huge threat to human health and places enormous economic burden to the society $[3,4]$. Studies indicated that prediabetes is a major factor leading to diabetes [5], and people with prediabetes have increased risk of cardiovascular disease (CVD) and mortality [6-8].

Given the potentially immerse disease burden caused by prediabetes, it is impending to implement cost-effective interventions to delay diabetes among subjects with prediabetes and even reverse the prediabetes status. Delivery of short message service (SMS) is part of mobile-based interventions that has bridged an effective communication channel to endeavor behavioral change, enhance disease-specific knowledge, and subsequently improve health outcomes in the field of preventive medicine and chronic disease self-management. Over the past decade, self-management education or support by mobile-based applications has been launched to target the blood glucose and $\mathrm{HbAlC}$ levels control in patients with type 2 diabetes mellitus (T2DM) [9]. Mobile-based applications are evident to modify the individual behavior to quit smoking [10], but they are rarely applied in diabetes prevention. A randomized controlled trial was conducted recently to evaluate the efficacy of the SMS intervention and it showed encouraging results in reducing T2DM onset among patients with impaired glucose tolerance (IGT) [11]. 
Regarding the cost-effectiveness analyses for add-on interventions conventionally applied to prediabetes, the prediabetic screening [12] and lifestyle modification [13-16] have been demonstrated to be highly cost-effective strategies in comparison with usual clinical practice. To determine whether a lifestyle modification is cost-effective, it depends on how preventive the specific strategy is and how much it costs. Although previous cost-effective strategies of lifestyle intervention have different combinations of interventions, they achieve cost-effectiveness outcomes by decreasing the incidence of T2DM and its complications through slowing the progression to T2DM and early detection of undiagnosed abnormal glucose tolerance [16-19].

In view of the significant effectiveness of the SMS intervention in preventing T2DM among prediabetes subjects and its relative low cost, it is tempting to assume that the SMS intervention is cost-effective. However, it is unethical and infeasible to wait until perfect lifetime data are available to validate the cost-effectiveness of any intervention. Decision modelling is proved to be an effective method to conduct economic evaluation of clinical interventions over lifetime. This technique compares the intervention of interest with the alternative options by incorporating all appropriate evidence of costs and effectiveness, simulating disease paths over longer time span, and reflecting uncertainty in evidence [20].

Using data from relevant epidemiological studies and clinical trials, we constructed a Markov model to compare the strategy of delivering the SMS intervention programme as an additional support to usual clinical practice and usual clinical practice alone in managing subjects with IGT. Depending upon the strategy applied, subjects in SMS intervention group had lower T2DM onset rate compared to the usual care, resulting in lower cost of managing T2DM and longer life years, which might compensate the additional cost of delivering the SMS intervention.

\section{Method}

2.1. Markov Model. A decision analytic model with a statetransition Markov process [21] was developed to simulate long-term effects of cost and clinical effectiveness of interventions in a cohort of prediabetes under two main strategies, which were (1) SMS intervention in addition to usual clinical practice and (2) usual clinical practice. The TreeAge Pro 2013 software (TreeAge Software, Inc., Williamstown, MA, USA) was used for the modelling. Long-term modelling referred to time horizon over a 50-year period beyond the twoyear intervention. The natural history referred to previous economic evaluation of diabetes prevention [15, 22-24]. The one-year transition cycle moved from one health state to another amongst four Markov states: normal glucose tolerance (NGT), IGT, T2DM, and death (Figure 1). An individual had the likelihood to transit from current health state to a different health state or remain in their current health state at the end of one-year cycle in this Markov process [21]. Compared to the usual practice group, the SMS intervention led to different transition probabilities among these four disease states, resulting in extra cost in addition to usual care. For patients who transit to diabetes were assumed to

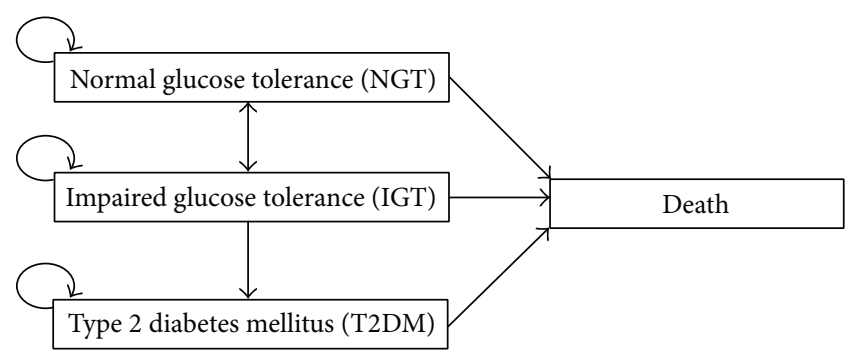

Figure 1: Annual transition diagram of Markov model.

stop receiving SMS intervention, therefore, the management of T2DM in these two groups was assumed to follow the same routine clinical practice in primary care setting. In other words, the costs and health effects of T2DM were the same for all subjects, regardless of which group they belonged to. An annual discount rate of $3 \%$ was undertaken in both the cost and health outcomes as per the Panel on Cost-Effectiveness in Health and Medicine recommended [25].

2.2. Transition Probability. As shown in Table 2, the annual transition probabilities between health states were taken from several data sources, including epidemiological studies and estimation from cost-effectiveness models. The annual transition probability from IGT to T2DM in the usual practice group in the first three years was adopted from the results of the placebo arm in Diabetes Prevention Program (DPP) [27], whereas the Diabetes Prevention Program Outcome Study (DPPOS) [28] provided this transition probability for the fourth year onwards. Subjects with IGT had about double risk for T2DM during the first three years compared to that in the fourth year onwards $[27,28]$. The effect of SMS on the transition from IGT to T2DM was reflected in the relative risk of T2DM onset for SMS intervention against control groups, which was reported by a randomized control trial (Clinical Trials Registry Number: NCT01556880) among a sample of Chinese professional drivers with IGT [11, 2931]. We adopted the relative risks for complete case analysis reported. This trial also reveals the drop-out rates of SMS intervention during the first year and second year, which were $38.9 \%$ and $30.3 \%$, respectively. The proportion of subjects who regressed to normal glucose regulation was taken from the Caro et al. study [22] which was used to derive the annual transition probabilities from DPP. Subjects with diagnosed T2DM would either stay in that state or be absorbed into the death state in the next year. All-cause mortality rates for NGT were adopted from the Hong Kong Life Table 2011 [32]. The relative risks of mortality in IGT and T2DM were $1.50(95 \%$ CI 1.10-2.00) and 2.30 (95\% CI 1.60-3.20), respectively, which were used to adjust the age-specific death rate for subjects with IGT or T2DM [23]. The incidence and mortality rates reported in literature were converted to annual transition probabilities using mathematical formula [33]: $p=1-$ $\exp (-r t)$, where $p$ is the transition probability, $r$ is the rate, and $t$ is the unit of time. Although subjects with diabetic complication had a higher risk of mortality, our model did not differentiate different complications related to diabetes and 
therefore applied the average mortality of DM for all diabetic subjects.

2.3. Costs. Costs were estimated from the perspective of health service provider. No clinical health service was deployed to routinely screen or treat patients with prediabetes in Hong Kong, and thus there were no costs assigned to patients with NGT or prediabetes in the usual practice group. The cost of a SMS intervention was the sum of two components: the delivery cost of a total of 66 text messages package via online programme platform and the staff cost of sending those text messages to each subject. The delivery cost of text messages per subject was $\$ 4.15$ in first year and $\$ 0.92$ in second year, in total of $\$ 5.08$, while the staff cost of sending text messages was estimated as $\$ 36.95$ based on an estimate of 5 minutes for delivering one SMS online and a median hourly wage of $\$ 6.72$ in 2011 [26]. The total intervention cost per subject was $\$ 42.03$. All costs were calculated in 2011 Hong Kong dollars and converted to US dollars at a pegged rate of US $\$ 1=$ HKD $\$ 7.8$. The annual total medical costs attributed to each patient with type 2 diabetes were $\$ 1492.05$ in 2004 year price [3], and those costs incurred in the usual clinical practice were inflated to $\$ 1,729.90$ in 2011 using the medical services price index taken from Hong Kong Census and Statistics Department [26]. The medical costs associated with diabetes management were assumed to be the same for all the years in this state. All costs data are summarized in Table 1.

2.4. Utilities. To calculate the quality adjusted life years (QALYs) accumulated in the lifetime model, we adopted the utility scores for each health state from literature. We applied the same utility scores for subjects with NGT and IGT. The utility scores for NGT/IGT and T2DM were 0.76 and 0.72 , respectively [23].

2.5. Cost-Effectiveness Outcomes. The main outcomes of the cost-effectiveness analysis in this study were the incremental cost-effectiveness ratios (ICER), in terms of cost per event (T2DM onset) prevented, cost per life year gained, and cost per QALY gained. The SMS intervention dominated control group if the control group was more expensive and less effective.

2.6. Sensitivity Analysis. Sensitivity analysis was performed to explore the uncertainty on the clinical and interventional parameters of model. Sensitivity analysis for the ICER of the SMS intervention compared with usual clinical practice was conducted on the transition probabilities with ranges suggested by previous literature and experts in family medicine and endocrine (Table 2). In addition, annual discount rate with limits bounded by $0 \%$ (undiscounted) and $5 \%$ was included in the analysis. Threshold analysis was undertaken to capture the threshold values of model interventional parameters at which the 50-year cumulative costs of SMS group were equivalent to those of control group. For instance, threshold values of intervention costs guided decision making on the level of intervention subsidy from the health service provider.

\section{Results}

3.1. Base-Case Scenario. The base-case scenario was based on the SMS intervention and T2DM costs from Table 1 and previously reported clinical parameters values from Table 2. Results of base-case scenario are shown in Table 3. During the two-year SMS intervention, compared to the usual practice group, each subject in SMS group costs $\$ 118.39$ less and the SMS group reduced $5.05 \%$ of T2DM onset. Over the 50 year period of time horizon, the cumulative costs of the SMS intervention group per subject were substantially lower than the control group. Given the 0.063 life years and 0.071 QALYs gained in SMS group, the SMS intervention was a less costly but more effective strategy than control group. As a result, the SMS intervention was beneficial for effectiveness and costsaving compared with the usual clinical practice in both short and long time horizons.

3.2. Sensitivity Analysis. Sensitivity analysis was performed by varying clinical and interventional parameters to test the robustness of model conclusion. Table 4 presents the results of sensitivity analysis which assessed the model robustness of base-case scenario. The determinant status of SMS group stayed unchanged when we varied various key parameters including SMS drop-out rates, annual transition probabilities between health states, and discount rate. Increased dropout rate at first year from base-case value to $100 \%$ led to the zero increment cost, remaining dominant. Despite variation in drop-out rates, the SMS intervention dominated control group. When the annual transition probability from IGT to T2DM at the first year of SMS intervention was increased from $3.53 \%$ to $11.57 \%$, which is equal to that in the control group, the SMS group still cost $\$ 76.21$ less, remaining dominant. The threshold analysis, as shown in Table 5, indicated that SMS group reached the equivalent amount of total costs of control groups when the first year intervention cost was increased to $\$ 1,704.04$ per person from original cost of $\$ 34.38$. When the first year intervention cost was fixed at $\$ 34.38$, to reach the same total cost as control group, the SMS intervention cost in the second year should have increased from $\$ 7.64$ to $\$ 3,093.78$. The annual transition probability from IGT to T2DM in first year in SMS group had to rise from $3.53 \%$ to $12.22 \%$ before the SMS group became no cheaper than control group. Similarly, only if the annual probability from IGT to T2DM in second year enrolled in SMS increased to $22.60 \%$ or higher, three times as base-case scenario, would their total medical cost be higher than the subjects in control group.

\section{Discussion}

This study found that, compared to usual clinical practice only, it was cost-saving to add the nonpharmacological SMS intervention in both the short and long time horizon. Given the high drop-out rate in the SMS group from the previous study, we assumed that the SMS intervention was provided only in the first two years. Within the two-year span, the SMS intervention led to a reduction of $5.05 \%$ of diabetes onset in comparison with the usual practice. Although it 
TABLE 1: Unit costs (US\$, year 2011 values) for the SMS intervention.

\begin{tabular}{|c|c|c|}
\hline Resource component & Unit cost & Reference \\
\hline \multicolumn{3}{|l|}{ SMS intervention } \\
\hline \multicolumn{3}{|l|}{ First year } \\
\hline Delivery charges of SMS via online platform* & $\$ 4.15$ & [11] \\
\hline Staff wage for SMS delivery ${ }^{\dagger}$ & $\$ 30.23$ & [26] \\
\hline \multicolumn{3}{|l|}{ Second year } \\
\hline Delivery charges of SMS via online platform* & $\$ 0.92$ & [11] \\
\hline Staff wage for SMS delivery ${ }^{\dagger}$ & $\$ 6.72$ & [26] \\
\hline Annual cost of T2DM $\mathrm{DM}^{\ddagger}$ & $\$ 1,727.90$ & [3] \\
\hline
\end{tabular}

TABLE 2: Clinical parameter value in base-case scenario and range used in sensitivity analysis.

\begin{tabular}{|c|c|c|c|c|}
\hline \multirow{2}{*}{ Parameter } & \multirow{2}{*}{ Base-case } & \multicolumn{2}{|c|}{ Sensitivity analysis } & \multirow{2}{*}{ Reference } \\
\hline & & Minimum & Maximum & \\
\hline \multicolumn{5}{|l|}{ SMS intervention } \\
\hline Drop-out rate at year 1 & $38.89 \%$ & $0 \%$ & $100 \%$ & [11] \\
\hline Drop-out rate at year 2 & $30.30 \%$ & $0 \%$ & $100 \%$ & {$[11]$} \\
\hline Relative risk of T2DM at year 1 from IGT & 0.34 & 0.10 & 1.00 & {$[11]$} \\
\hline Relative risk of T2DM at year 2 from IGT & 0.60 & 0.10 & 1.00 & [11] \\
\hline \multicolumn{5}{|l|}{ Annual transition probability from IGT to NGT } \\
\hline At all years & $16.20 \%$ & $5 \%$ & $25 \%$ & {$[22]$} \\
\hline \multicolumn{5}{|l|}{ Annual transition probability from NGT to IGT } \\
\hline At all years & $16.30 \%$ & $5 \%$ & $25 \%$ & {$[22]$} \\
\hline \multicolumn{5}{|c|}{ Incidence rate (cases per 100 person-years) of T2DM from IGT } \\
\hline Control at years $1-3$ & 11.0 & 9.8 & 12.3 & {$[27]$} \\
\hline Control at year $4+$ & 5.6 & 4.8 & 6.5 & {$[28]$} \\
\hline \multicolumn{5}{|l|}{ Relative risk of mortality } \\
\hline IGT & 1.5 & 1.1 & 2 & {$[23]$} \\
\hline T2DM & 2.3 & 1.6 & 3.2 & {$[23]$} \\
\hline \multicolumn{5}{|l|}{ Utility } \\
\hline NGT & 0.76 & \multicolumn{2}{|c|}{ NA } & {$[23]$} \\
\hline IGT & 0.76 & \multicolumn{2}{|c|}{ NA } & {$[23]$} \\
\hline T2DM & 0.72 & \multicolumn{2}{|c|}{ NA } & {$[23]$} \\
\hline Discount rate & $3 \%$ & $0 \%$ & $5 \%$ & {$[25]$} \\
\hline
\end{tabular}

Note: NGT = normal glucose tolerance; IGT = impaired glucose tolerance; T2DM = type 2 diabetes mellitus; NA = not applicable.

TABLE 3: Results of base-case scenario.

\begin{tabular}{lccc}
\hline Base-case scenario & SMS & Control & Incremental \\
\hline 2-year period & & & -118.39 \\
Mean cost (in USD) accrued per patient & 342.94 & $-5.05 \%$ & Dominance \\
T2DM onset & $12.55 \%$ & $17.60 \%$ & -830.77 \\
Cost per T2DM onset prevented & & & 0.063 \\
50-year period & 12107.40 & 12958.17 & 0.071 \\
Mean cost (in USD) accrued per patient & 19.24 & 14.08 & Dominance \\
LYs per patient & 14.248 & & Dominance \\
QALYs per patient & & & \\
Cost per LY gained & & & \\
Cost per QALYs gained & & & \\
\hline
\end{tabular}

Note: T2DM = type 2 diabetes; LYs = life years; QALY = quality adjusted life year. 
TABLE 4: Results of sensitivity analyses.

\begin{tabular}{lcccc}
\hline Parameters & Base-case & $\begin{array}{c}\text { Range for sensitivity } \\
\text { analysis }\end{array}$ & $\begin{array}{c}\text { Range for incremental } \\
\text { cost (USD) }\end{array}$ & $\begin{array}{c}\text { Range for cost } \\
\text { per LYs gained }\end{array}$ \\
\hline SMS drop-out rate at year 1 & $38.89 \%$ & $0.00 \%-100.00 \%$ & -1669.66 to 0.00 & Dominance \\
SMS drop-out rate at year 2 & $30.30 \%$ & $0.00 \%-100.00 \%$ & -1131.28 to -765.21 & Dominance \\
Annual transition probability & & & -950.73 to -761.82 & Dominance \\
From IGT to T2DM, control at year 1-3 & $10.42 \%$ & $9.34 \%-11.57 \%$ & -1324.36 to -76.21 & Dominance \\
From IGT to T2DM, SMS at year 1 & $3.53 \%$ & $0.93 \%-11.57 \%$ & -1352.05 to -688.20 & Dominance \\
From IGT to T2DM, SMS at year 2 & $6.25 \%$ & $0.93 \%-11.57 \%$ & -922.28 to -1063.06 & Dominance \\
From IGT to NGT & $16.20 \%$ & $5.00 \%-25.00 \%$ & -1117.45 to -979.19 & Dominance \\
From NGT to IGT & $16.30 \%$ & $5.00 \%-25.00 \%$ & -1015.49 to -1026.04 & Dominance \\
RR of mortality in IGT & 1.5 & $1.1-2.0$ & -1087.30 to -953.95 & Dominance \\
RR of mortality in T2DM & 2.3 & $1.6-3.2$ & -1450.41 to -836.95 & Dominance \\
Discount rate & $3.00 \%$ & $0.00 \%-5.00 \%$ &
\end{tabular}

Note: $\mathrm{NGT}$ = normal glucose tolerance; IGT = impaired glucose tolerance; T2DM = type 2 diabetes mellitus; RR = relative risk.

TABLE 5: Threshold analysis of parameters at which the costs of SMS intervention and control became equivalent over a 50-year period.

\begin{tabular}{lcc}
\hline Parameters & Base-case & Threshold \\
\hline SMS intervention cost at year 1 & $\$ 34.38$ & $\$ 1,704.04$ \\
SMS intervention cost at year 2 & $\$ 7.64$ & $\$ 3,093.78$ \\
Annual transition probability & & \\
$\quad$ From IGT to T2DM, SMS at year 1 & $3.53 \%$ & $12.22 \%$ \\
$\quad$ From IGT to T2DM, SMS at year 2 & $6.25 \%$ & $22.60 \%$ \\
\hline
\end{tabular}

Note: IGT = impaired glucose tolerance; T2DM = type 2 diabetes mellitus.

costs a total of $\$ 42.03$ per subject to conduct the SMS intervention for two years, the money saved by treating less diabetic cases overcompensated the cost of SMS intervention. As a result, the SMS group cost $\$ 118.39$ less per subject and prevented $5.05 \%$ of diabetes onset. When projecting the effects of the discrepancy in DM onset between the two groups to the lifetime span, we found that the SMS intervention accrued more life years gained (incremental effectiveness: 0.071 QALY) and less cost (incremental cost: $-\$ 1,020.35)$ than the control group. The robustness of the lifetime model was verified by sensitivity analysis through varying the transition probabilities and drop-out within wide ranges. The threshold analysis showed that only when the cost of SMS intervention climbed to about $\$ 2000$ or more to reach the break-even point with the control group.

While the SMS intervention has been shown to be costsaving in prediabetic population, the ICER values were not available as the incremental cost is negative. Using the empirical DPP/DPPOS data [16], the 10-year cost-effectiveness analysis estimated that metformin overwhelmed the control group with direct medical cost of care, and the ICER for lifestyle intervention compared to control group was $\$ 10,037$ per QALYs gained. Complementary results were reported in Australian study modelling from a third-party payer perspective [23], indicating that the intensive lifestyle modification dominated the control group but the ICER for the metformin versus control group was $\$ 10,142$ in Australian dollars per QALYs gained. Lifestyle interventions in IGT were found cost-effective in other contexts as well, while most of these studies also adopted the effectiveness data from the DPP/DPPOS cohort $[22,34,35]$. Some studies on the CEA of interventions in prediabetes have established more sophisticated models, which involve development of complications after subjects developed diabetes. These studies adopted the Framingham or UKPDS risk functions to differentiate the risks of diabetic complications in long term based on the changes of clinical parameters (i.e., HbAlc, lipid profile, systolic blood pressure, etc.) observed in the trials. The incremental effectiveness in these studies is more prominent than the finding in our study that 0.14 to 0.50 life years were gained by lifestyle intervention compared to no intervention over the lifetime [14, 18]. Although the incremental life years and QALYs found in our model were smaller, it is noteworthy that we made a rather conservative estimation, as we assumed the two intervention groups have the same utilities after they developed diabetes. Moreover, we found that the SMS intervention is cost-saving over both short term and long term, which is favouring results for policy makers.

The main strength of this cost-effectiveness analysis model was that the sources of interventional parameter were the valid estimate reported in a randomized controlled trial. Additionally, our model estimated the short-term as well as long-term outcomes of cost-effectiveness. This is important as the initial setup cost invested to SMS group was shortly balanced out within two years, and the incremental cost was proportionally magnified between the two groups over a 50-year period. As the threshold analysis supported the increased costs of SMS in first and second year, there is potential area to set the patients' out-of-pocket charges of SMS intervention implemented in health service. Subsidy given by health service provider or government may be an alternative approach to deal with financing of the SMS implementation. Threshold analysis further offered cautionary advice on the termination of SMS intervention when the annual transition probabilities from IGT to T2DM in first and second year of 
SMS group exceeded $12.22 \%$ and $22.60 \%$, respectively. The scenarios implied that the SMS intervention was no longer supported by the nature of cost-saving. The feasibility and sustainability of SMS intervention are another concern due to about one-third of drop-out rates in first and second year. Owing to the high levels of drop-out, the effects of SMS intervention on ICER were considerably diluted. In a scenario of $100 \%$ drop-out in second year participation as illustrated in sensitivity analysis, the incremental cost (\$765.21) of one-year intervention was smaller than that in base-case scenario.

Several drawbacks were noted in this study. Firstly, the effectiveness of SMS intervention was based on a RCT in Hong Kong Chinese population, but some clinical and epidemiological data were adopted from DPP and DPPOS, which are the US population-based studies. Due to lack of Hong Kong local data, we assumed that data from other sources were applicable in our model. It is likely that there may be substantial differences in the clinical and epidemiological data between the Hong Kong and the US populations; however, the values of these parameters were applied the same in both SMS group and control group, which will not affect the relative effectiveness in the model. Secondly, the interventional parameters were derived from randomized controlled trial on the pilot basis with 104 IGT subjects participating. Although the intervention has been shown to be effective in pilot data, the effect of SMS on the reduction of T2DM onset over time may be diluted in populationbased setting. This study reflects the reasonable need for undertaking the SMS intervention on mass population with prediabetes. Thirdly, the model was built based on some simplified assumptions. For example, annual cost of T2DM was uniform regardless of gender, complication experienced, and insulin treated. Given the more diabetic complications experienced, direct medical costs associated with a T2DM subject increased sharply [36]. Furthermore, the model did not account for the health states representing the presence of diabetic complications as no evidence available shows that the SMS intervention in prediabetes has impacts on the incidence of diabetic complications.

\section{Conclusions}

This cost-effectiveness analysis reveals that the SMS intervention for subjects with prediabetes had the superiority of lower cost and a considerable improvement in preventing or delaying the T2DM onset. Encouraging efforts of clinical and cost-effectiveness outcomes were diluted due to the loss of participation over the 2-year intervention. This study indicated that it was cost-saving to prevent T2DM through implementing nonpharmacological SMS intervention among prediabetics.

\section{The Significant Findings of the Study}

The SMS intervention was a low-cost and effective programme for type 2 diabetes mellitus prevention in subjects with impaired glucose tolerance, resulting in cost-saving to health service provider regardless of 2-year trial and 50-year lifetime periods.

\section{What This Study Adds}

This trial-based cost-effectiveness analysis showed that the SMS may be an add-on intervention applied to impaired glucose tolerance management in routine clinical practice in primary care setting.

\section{Abbreviations \\ SMS: $\quad$ Short message service \\ T2DM: Type 2 diabetes mellitus \\ IGT: Impaired glucose tolerance \\ QALY: Quality adjusted life year \\ DPP: Diabetes Prevention Program \\ DPPOS: Diabetes Prevention Program Outcome Study \\ ICER: Incremental cost-effectiveness ratios.}

\section{Conflict of Interests}

The authors declare that they have no competing interests.

\section{Authors' Contribution}

Carlos K. H. Wong and Fang-Fang Jiao provided direct input into the design and execution of the study. Carlos K. H. Wong, Fang-Fang Jiao, and Daniel Y. T. Fong undertook statistical analysis and generated the results. Carlos K. H. Wong, ShingChung Siu, Fang-Fang Jiao, Daniel Y. T. Fong, Ka-Wai Wong, Esther Y. T. Yu, and Yvonne Y. C. Lo provided input to data interpretation. Carlos K. H. Wong drafted the paper. All authors contributed to its editing. All authors read and approved the final paper.

\section{Acknowledgment}

The earlier work that provided the interventional parameters of this model was kindly supported by the Board of the Tung Wah Group of Hospitals, Hong Kong. The article processing charge of this paper was supported by Tung Wah Eastern Hospital.

\section{References}

[1] L. Guariguata, D. R. Whiting, I. Hambleton, J. Beagley, U. Linnenkamp, and J. E. Shaw, "Global estimates of diabetes prevalence for 2013 and projections for 2035," Diabetes Research and Clinical Practice, vol. 103, no. 2, pp. 137-149, 2014.

[2] UK Prospective Diabetes Study (UKPDS) Group, "Intensive blood-glucose control with sulphonylureas or insulin compared with conventional treatment and risk of complications in patients with type 2 diabetes (UKPDS 33)," The Lancet, vol. 352, no. 9131, pp. 837-853, 1998.

[3] B. S. W. Chan, M. W. Tsang, V. W. Y. Lee, and K. K. C. Lee, "Cost of type 2 diabetes mellitus in Hong Kong Chinese," International Journal of Clinical Pharmacology and Therapeutics, vol. 45, no. 8, pp. 455-468, 2007.

[4] American Diabetes Association, "Economic costs of diabetes in the U.S. in 2012,” Diabetes Care, vol. 36, pp. 1033-1046, 2013. 
[5] D. Noble, R. Mathur, T. Dent, C. Meads, and T. Greenhalgh, "Risk models and scores for type 2 diabetes: systematic review," British Medical Journal, vol. 343, Article ID d7163, 2011.

[6] Y. S. Levitzky, M. J. Pencina, R. B. D’Agostino et al., "Impact of impaired fasting glucose on cardiovascular disease: the framingham heart study," Journal of the American College of Cardiology, vol. 51, no. 3, pp. 264-270, 2008.

[7] E. Selvin, M. W. Steffes, H. Zhu et al., "Glycated hemoglobin, diabetes, and cardiovascular risk in nondiabetic adults," The New England Journal of Medicine, vol. 362, no. 9, pp. 800-811, 2010.

[8] S. Seshasai, S. Kaptoge, A. Thompson et al., "Diabetes mellitus, fasting glucose, and risk of cause-specific death," The New England Journal of Medicine, vol. 364, no. 9, pp. 829-841, 2011.

[9] S. Krishna and S. A. Boren, "Diabetes self-management care via cell phone: a systematic review," Journal of Diabetes Science and Technology, vol. 2, no. 3, pp. 509-517, 2008.

[10] C. Free, R. Knight, S. Robertson et al., "Smoking cessation support delivered via mobile phone text messaging (txt2stop): a single-blind, randomised trial," The Lancet, vol. 378, no. 9785, pp. 49-55, 2011.

[11] C. K. H. Wong, C. S. C. Fung, S. C. Siu et al., "A short message service (SMS) intervention to prevent diabetes in Chinese professional drivers with pre-diabetes: a pilot single-blinded randomized controlled trial," Diabetes Research and Clinical Practice, vol. 102, no. 3, pp. 158-166, 2013.

[12] R. Chatterjee, K. M. V. Narayan, J. Lipscomb, and L. S. Phillips, "Screening adults for pre-diabetes and diabetes may be costsaving," Diabetes Care, vol. 33, no. 7, pp. 1484-1490, 2010.

[13] M. K. Eriksson, L. Hagberg, L. Lindholm, E.-B. MalmgrenOlsson, J. Österlind, and M. Eliasson, "Quality of life and costeffectiveness of a 3-year trial of lifestyle intervention in primary health care," Archives of Internal Medicine, vol. 170, no. 16, pp. 1470-1479, 2010.

[14] P. Lindgren, J. Lindström, J. Tuomilehto et al., "Lifestyle intervention to prevent diabetes in men and women with impaired glucose tolerance is cost-effective," International Journal of Technology Assessment in Health Care, vol. 23, no. 2, pp. 177-183, 2007.

[15] A. Neumann, P. Schwarz, and L. Lindholm, "Estimating the cost-effectiveness of lifestyle intervention programmes to prevent diabetes based on an example from Germany: Markov modelling," Cost Effectiveness and Resource Allocation, vol. 9, article 17, 2011.

[16] Diabetes Prevention Program Research Group, “The 10year cost-effectiveness of lifestyle intervention or metformin for diabetes prevention: an intent-to-treat analysis of the DPP/DPPOS," Diabetes Care, vol. 35, no. 4, pp. 723-730, 2012.

[17] C. L. Gillies, P. C. Lambert, K. R. Abrams et al., "Different strategies for screening and prevention of type 2 diabetes in adults: cost effectiveness analysis," The BMJ, vol. 336, no. 7654, pp. 1180-1184, 2008.

[18] W. H. Herman, T. J. Hoerger, M. Brandle et al., "The costeffectiveness of lifestyle modification or metformin in preventing type 2 diabetes in adults with impaired glucose tolerance," Annals of Internal Medicine, vol. 142, no. 5, pp. 323-332, 2005.

[19] T. J. Hoerger, K. A. Hicks, S. W. Sorensen et al., "Costeffectiveness of screening for pre-diabetes among overweight and obese U.S. Adults," Diabetes Care, vol. 30, no. 11, pp. 28742879, 2007.
[20] A. Briggs, K. Claxton, and M. Sculpher, Decision Modelling for Health Economic Evaluation, Oxford University Press, Oxford, UK, 2006.

[21] F. A. Sonnenberg and J. R. Beck, "Markov models in medical decision making: a practical guide," Medical Decision Making, vol. 13, no. 4, pp. 322-338, 1993.

[22] J. J. Caro, D. Getsios, I. Caro, W. S. Klittich, and J. A. O’Brien, "Economic evaluation of therapeutic interventions to prevent type 2 diabetes in Canada," Diabetic Medicine, vol. 21, no. 11, pp. 1229-1236, 2004.

[23] A. J. Palmer and D. M. D. Tucker, "Cost and clinical implications of diabetes prevention in an Australian setting: a long-term modeling analysis," Primary Care Diabetes, vol. 6, no. 2, pp. 109121, 2012.

[24] K. Dalziel and L. Segal, "Time to give nutrition interventions a higher profile: cost-effectiveness of 10 nutrition interventions," Health Promotion International, vol. 22, no. 4, pp. 271-283, 2007.

[25] J. E. Siegel, M. C. Weinstein, L. B. Russell, and M. R. Gold, "Recommendations for reporting cost-effectiveness analyses," Journal of the American Medical Association, vol. 276, no. 16, pp. 1339-1341, 1996.

[26] Census and Statistics Department. Hong Kong Special Administrative Region, Hong Kong Annual Digest of Statistics 2012, 2012.

[27] W. C. Knowler, E. Barrett-Connor, S. E. Fowler et al., "Reduction in the incidence of type 2 diabetes with lifestyle intervention or metformin," The New England Journal of Medicine, vol. 346, no. 6, pp. 393-403, 2002.

[28] Diabetes Prevention Program Research Group, "10-year followup of diabetes incidence and weight loss in the Diabetes Prevention Program Outcomes Study," The Lancet, vol. 374, no. 9702, pp. 1677-1686, 2009.

[29] S. C. Siu, K. W. Wong, K. F. Lee et al., "Prevalence of undiagnosed diabetes mellitus and cardiovascular risk factors in Hong Kong professional drivers," Diabetes Research and Clinical Practice, vol. 96, no. 1, pp. 60-67, 2012.

[30] C. K. H. Wong, C. S. C. Fung, S.-C. Siu et al., "The impact of work nature, lifestyle, and obesity on health-related quality of life in Chinese professional drivers," Journal of Occupational and Environmental Medicine, vol. 54, no. 8, pp. 989-994, 2012.

[31] C. K. Wong, S. C. Siu, E. Y. Wan et al., "Simple non-laboratoryand laboratory-based risk assessment algorithms and nomogram for detecting undiagnosed diabetes mellitus," Journal of Diabetes, 2015.

[32] Census and Statistics Department, Hong Kong Special Administrative Region. Hong Kong Life Tables 2006-2014, 2012.

[33] R. L. Fleurence and C. S. Hollenbeak, "Rates and probabilities in economic modelling: transformation, translation and appropriate application," PharmacoEconomics, vol. 25, no. 1, pp. 3-6, 2007.

[34] P. Watson, L. Preston, H. Squires, J. Chilcott, and A. Brennan, "Modelling the economics of type 2 diabetes mellitus prevention: a literature review of methods," Applied Health Economics and Health Policy, vol. 12, no. 3, pp. 239-253, 2014.

[35] A. J. Palmer, S. Roze, W. J. Valentine, G. A. Spinas, J. E. Shaw, and P. Z. Zimmet, "Intensive lifestyle changes or metformin in patients with impaired glucose tolerance: modeling the longterm health economic implications of the diabetes prevention program in Australia, France, Germany, Switzerland, and the United Kingdom," Clinical Therapeutics, vol. 26, no. 2, pp. 304321, 2004. 
[36] M. Brandle, H. Zhou, B. R. K. Smith et al., "The direct medical cost of type 2 diabetes," Diabetes Care, vol. 26, no. 8, pp. 23002304, 2003. 


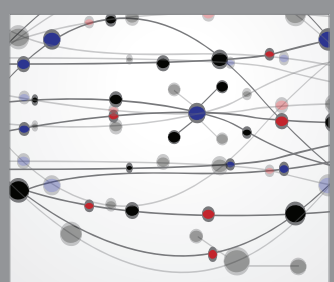

The Scientific World Journal
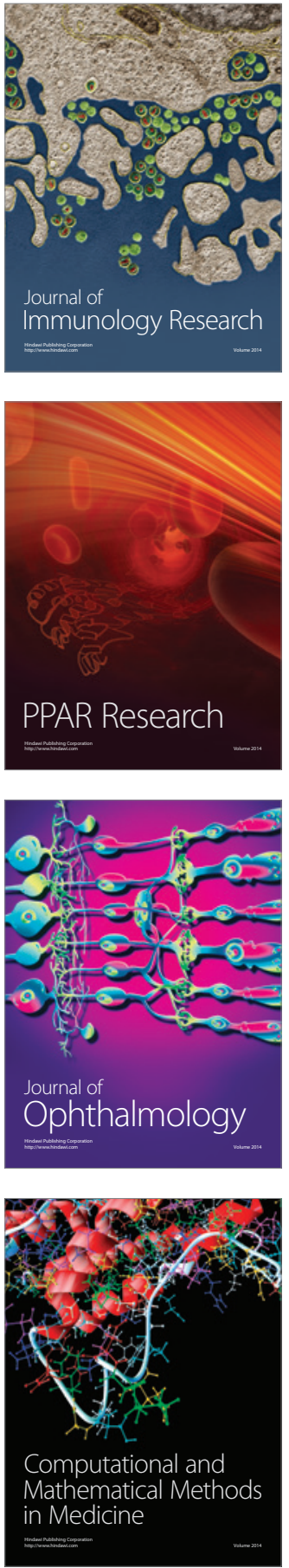

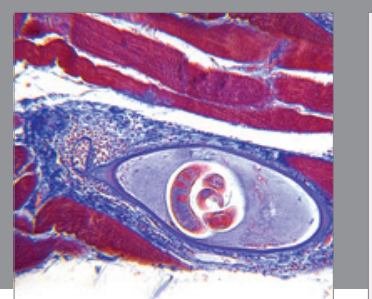

Gastroenterology Research and Practice

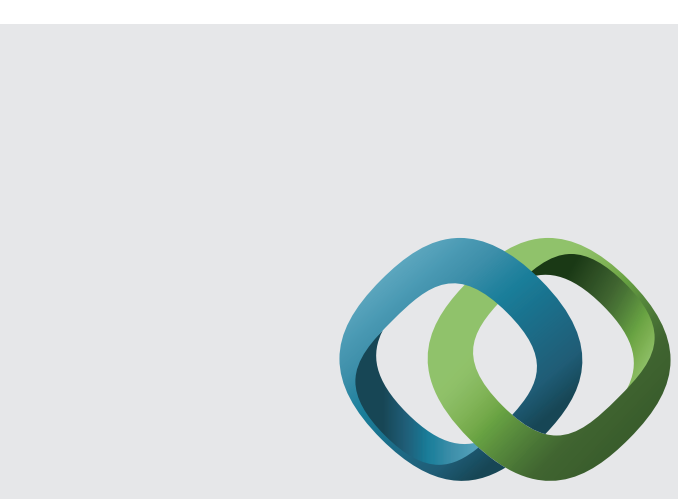

\section{Hindawi}

Submit your manuscripts at

http://www.hindawi.com
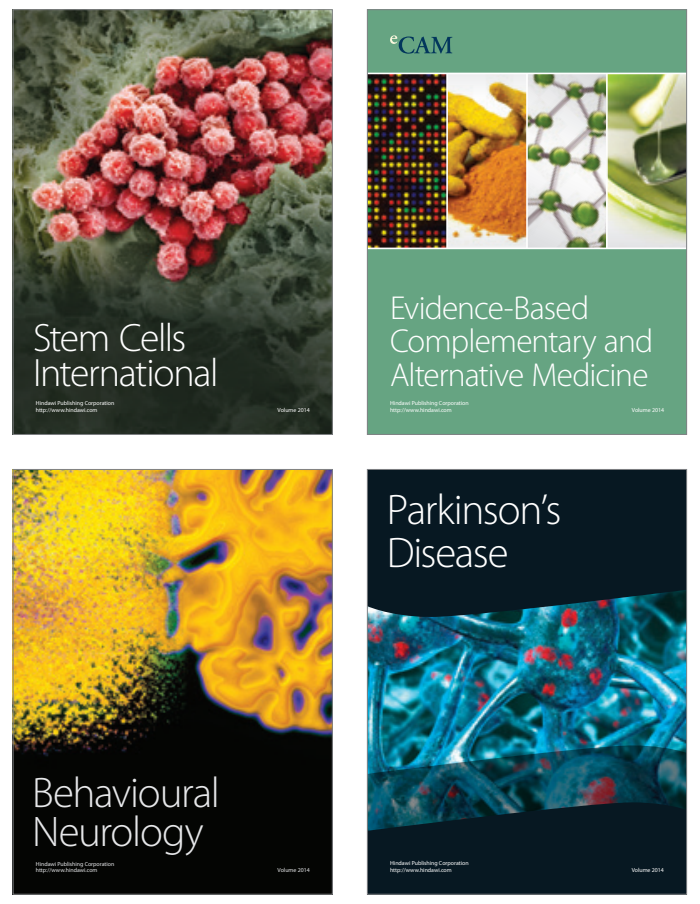
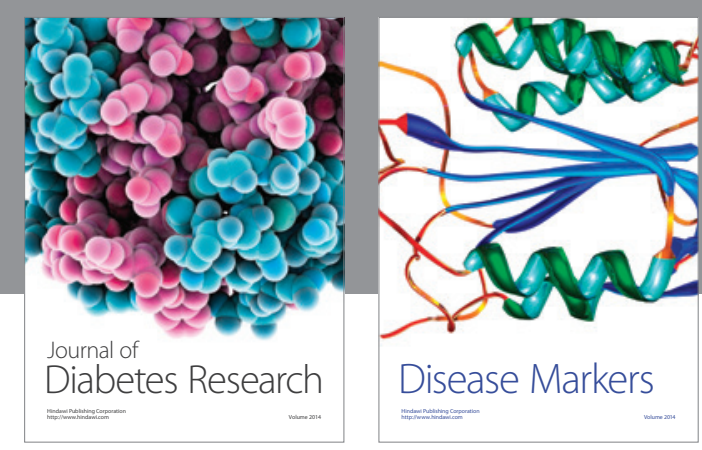

Disease Markers
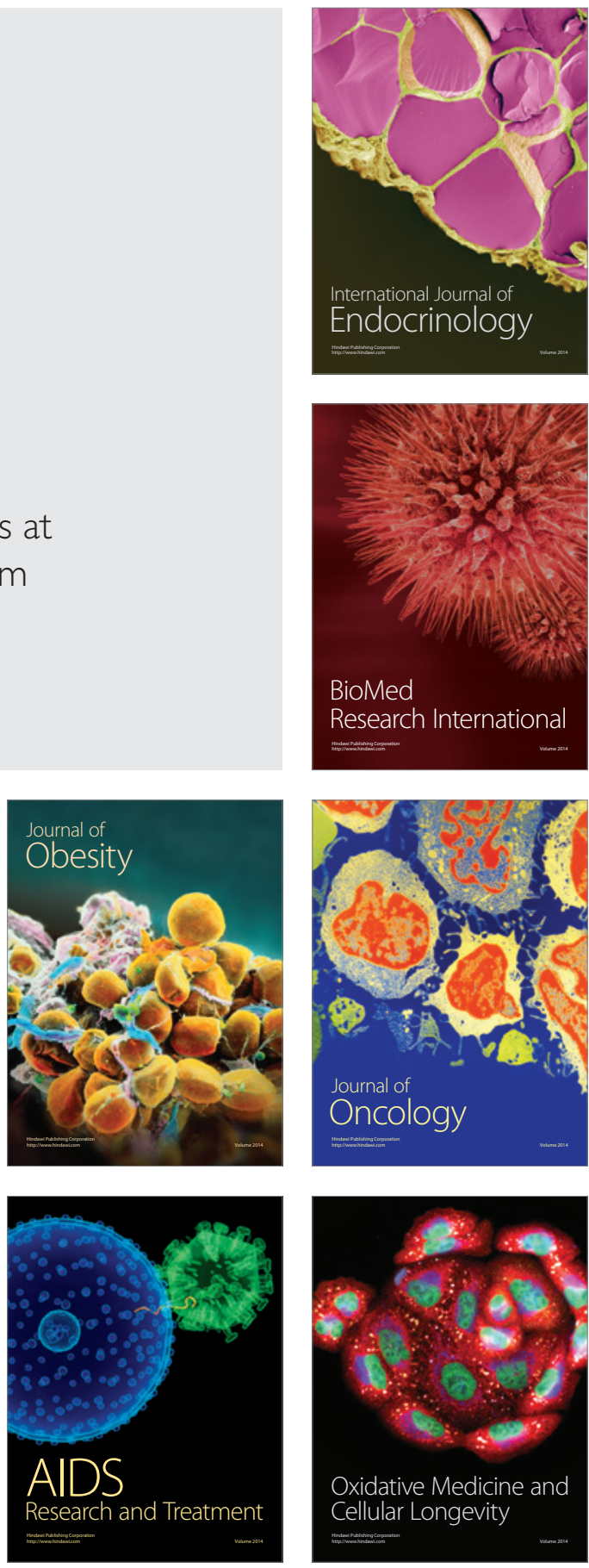